\title{
Exogenous Natural Glycoprotein Multiple Mechanisms of Anti-tumor Activity
}

\author{
Hong-Liang Yuan ${ }^{1,2 *}$, Xiao-Lei Liu ${ }^{3}$, Qi-Chang Dai ${ }^{1}$, Hui Song ${ }^{1,2}$
}

\begin{abstract}
Natural glycoproteins can induce apoptosis of tumor cells and exert anti-tumor activity by immunomodulatory functions, cytotoxic and anti-inflammation effects, and inhibition of endothelial growth factor. Given their prospects as novel agents, sources of natural antitumor glycoproteins have attracted attention and new research directions in glycoprotein biology are gradually shifting to the direction of cancer treatment and prevention of neoplastic disease. In this review, we summarize the latest findings with regard to the tumor suppressor signature of glycoproteins and underlying regulatory mechanisms.
\end{abstract}

Keywords: Glycoprotein - antitumor - immunomodulatory - cytotoxicity - antiinflammation - endothelial growth factor

Asian Pac J Cancer Prev, 16 (4), 1331-1336

\section{Introduction}

Glycoprotein for protein and sugar chain of covalent compounds, the glycoprotein in sort is more, widely distributed in animals, plants, and microorganisms, with free or combined with state widely exists in the cells inside and outside. Glycoprotein sugar chains have a high degree of heterogeneity. Under normal circumstances, the proportion of sugar is small compared with the protein fraction. Saccharides and glycoproteins interacting proteins involved in many physiological and pathological processes in vivo protein is the main bearer of its physiological function, sugar chains on protein function from the modification. Protein glycosylation is one of the most important post-translation modifications, many protein function are closely related to the realization of glycosylation, the sugar chain and protein interactions involved in cell transformation and differentiation, immune response, signal transduction, and other kinds of regulation and expression of life phenomena (Pandurangan et al., 2012).

Natural glycoprotein exists in plant cell wall, cell membrane and cytoplasm, including many enzymes, lectin, structure and storage proteins and receptors, in plant seed germination, seedling growth, reproduction and stress plays an important role in the process of life. Plant glycoprotein in a variety of tumor cells, in particular, affect their accept outside signal substance and cell information exchange with the outside world, immune regulation, the occurrence of cancer development, host- pathogen interaction, etc., make it widely used on antitumor activity (Grigorian et al., 2009). Glycoprotein are important physiological active substances, it involved in cell adhesion, tumor metastasis, cell growth process, and have enhanced immune regulation, inhibiting tumor, reduce blood sugar, blood fat, anti-oxidation, anti-aging, anti-tumor application research in recent years has made important progress in (Takahashi et al., 2009). The author only on the antitumor mechanism of natural glycoprotein and foreign research status were reviewed, which provided reference for related research.

\section{Natural Glycoprotein Immunomodulatory Anti-Tumor Effect}

Natural glycoprotein can by acting on the immune adjustment mechanisms to prevent cancer and anti-tumor effects. Natural glycoprotein role through a variety of immune system responses and control a variety of immune cell signal transduction, activate T cells, B cells and NK cell immune reaction channels, natural glycoprotein enhanced immune response, inhibit the growth of a wide variety of tumor cells and metastasis (Grigorian et al., 2009). In recent years, the ability of such tumor activity of native glycoprotein inhibition has been extensively studied. Neem leaf extracted from plants neem leaf glycoprotein (NLGP) is a kind of effective immune modulators, related immune mediated immune activation and polarization. NLGP activation of the early signs of CD69 lymphocytes, monocytes and increases the activity of dendritic cells, activate CD45RO, at the same time reduce CD45RA phenotype and CD62L expression. NLGP activation of 
$\mathrm{T}$ cells to secrete more $\mathrm{T}$ cofactor Th1, lower cytokine interferon gamma and Th2 cytokines interleukins IL-4. NLGP mediated immune activation and related immune response is NLGP can effectively through the increase in type I expressed in the immune response inhibition, can significantly protect host antitumor function and maintain normal immune steady (Bose et al., 2009).

Neem leaf glycoprotein laryngeal tumor cells can be induced cracking SLTCL immunomodulatory M2 macrophages in vitro, the activity of cytotoxic $\mathrm{T}$ cells and the effect of inhibiting tumor protein increased regulation of $\mathrm{T}$ cell (Goswami et al., 2014), and can be adjusted in cervical cancer patients with stage IIIB enzyme indoleamine 2, 3 two-plus oxygen content of dendritic cells in the tolerance, regulatory $\mathrm{T}$ cells in the enzyme indoleamine 2, 3 two-plus oxygen induction and activity of regulatory $\mathrm{T}$ cells is an important sign of cancer, neem leaf glycoprotein may inhibit induced enzyme indoleamine 2, 3 two-plus oxygen tolerance of dendritic cells by inhibiting the activity of regulatory $\mathrm{T}$ cells play a role of anti-tumor (Roy et al., 2013). Through the neem leaf glycoprotein of tumor microenvironment (TME) treatment in mice (NLGP-ME) research results show that the plant neem leaf glycoprotein has obvious inhibit the growth of mice B16 melanoma, improves the treatment ability of the survival of the mice, the inhibitory effect of B16 melanoma is directly and inhibiting tumor cell function of CD8+ cytotoxic T cell activity and cut about (Barik et al., 2013).

Neem leaf glycoprotein has no effect on immune cell apoptosis, but it can be induced from mice and rats mononuclear cell proliferation, increase hemoglobin levels, white blood cell count and the number of lymphocytes. The mice treated neem leaf glycoprotein total IgG levels increase; especially the IgG2a content also will increase (Das et al., 2014). By cancer immunotherapy found carcinoembryonic antigen (CEA) and colon cancer, lung cancer, carcinoma pathogenesis related, such as breast cancer research through integration of neem leaf glycoprotein immune mice spleen cells and non secretory myeloma cells to produce monoclonal antibody $1 \mathrm{C} 8,1 \mathrm{C} 8$ specificity to human CEA (Colo-205, HCT-205 and HT29) and (CT-26) in mice colon cancer cells, but with the CEA (MCF7, SiHa and SCC084) and rat (B16MelF10) cancer cells combined with low reaction. This $1 \mathrm{C} 8$ monoclonal antibody against neem leaf glycoprotein with in mice (CT)-26 in BALB/c mice and human (Colo-205) in the absence of thymus nude mice CEA cancer cells in the body, has significant antitumor activity and better ability to survive, to cell proliferation and cell migration of carcinoembryonic antigen without direct impact, by NK cells dependence strong antibody, rely on the cytotoxic effect of carcinoembryonic antigen cells and angiogenesis is the main antitumor (Das et al., 2014).

A glycoprotein isolated from green tea with a molecular weight $126.513 \mathrm{Da}$, the Tea glycoprotein can increase macrophage NO, secretion of cytokines reach immunomodulatory effects. NO is an endogenous vascular contraction Zhang factor, is activated macrophages to kill pathogenic microorganisms main effect factor, with the balance to maintain the cardiovascular system and prevent atherosclerosis role of macrophages in addition to a secretion of inflammatory cytokines NO outside the role, but also through the secretion of cytokines, play inflammation, anti-tumor immune response (Nie et al., 2008). The anti-oxidative and anticancer activities of tea glycoprotein (TGP) in green tea were studied shown that the TGP had a unique antioxidant activity. Furthermore, the anticancer activity of the TGP at different concentrations was evaluated by the MTT assay using two kinds of colon cancer cell lines HCT-15 and Caco-2. Dose-dependently TGP exhibited good anti-proliferation activity to HCT-15, whereas exhibited very weak anti-proliferation activity to Caco-2. The TGP obviously inhibited the proliferation of Caco-2 (Nie et al., 2007).

Pulsatilla glycoproteins isolated from Pulsatilla Chinensis (Bunge) Regel. of the water treated with the extract of purified homogeneous glycoprotein component, and a molecular weight of $62.5 \mathrm{kD}$. Significantly enhanced in vitro murine peritoneal macrophages neutral red effect, and can induce giant macrophages to produce nitric oxide, macrophages secrete interleukin-1 is also certain to enhance the role. Pulsatilla glycoprotein showed on mouse peritoneal macrophages in immune enhancement (Dai et al., 2000).

A glycoprotein extracted from Olden-landia diffusa (Willd1) Roxb1 was mainly a 90kDa glycoprotein. The Diffusa glycoprotein promoted proliferation of mouse spleen cells has a strong effectively, and enhanced antimouse and human cell-specific cytotoxic activity against tumor cells, enhanced B cells cytokine production of antibodies and monocytes generates and monocyte phagocytosis of tumor cells. The glycoprotein has immune adjustment function of mice and men, and by stimulating the body's immune system damage or tumor cells (Gupta et al., 2004; Wu et al., 2009).

Isolated from ZhiKong scallops a Glycoprotein (Glycoprotein of Chlamys farreri, GCF), GCF total protein content was $60 \%$, total sugar content was $36 \%$. Experimental results show that the GCF could significantly inhibit the growth of portability S180 sarcoma in tumorbring mice, the drug dose of $40 \mathrm{mg} / \mathrm{kg}$ effect best, inhibitory rate can reach $47.29 \%$. In addition, GCF also can obviously increase the tumor-burdened the weight of immune organs in mice, and could make a tumor-burdened mice abdominal immune cell activity restoration, raise a tumor-burdened the phagocytosis of macrophages in mice, can significantly improve a tumor-burdened NK cell activity in mice (Gu et al., 2001).

\section{Glycoprotein Inhibit Tumor Inflammation}

Inflammation and infection increase the risk of ovarian cancer (Mantovani et al., 2008). Inflammation has been found to be one of the critical processes that govern cancer biology following experiments showing that inflammatory cells, cytokines and chemokines contribute to tumour growth and progression (Hanahan et al., 2000). Consequently, non-steroidal anti-inflammatory drugs reduce the risk of cancer, decrease cell growth, induce apoptosis and inhibit the survival of cancer cells (Andrews et al., 2008). 
Cancer often appears in the process of inflammation in the ending progress, not in the starting stage. So there is the complex interaction between host immune cells in the process of the development of tumor, promote cancer and ability to limit or eliminate the occurrence and development of modern pathology definition around tumor inflammation and inflammatory cells: yes, it is an important marker of pathology, and with in the process of tumorigenesis, development and metastasis have inflammation and inflammatory cells produced in (Sandra et al., 2010). Plant glycoprotein associated can inhibit the inflammation factor epoxy synthase cox-2, induced nitric oxide synthase of iNOS and interleukins IL-1 beta, blocking the NF- $x \mathrm{~B}$ and MAPKs signals inflammation inhibiting tumor and metastasis (Lee et al., 2012). Isolated from benzoin belongs to wild jasmine a $38 \mathrm{kda}$ glycoprotein, the glycoprotein increase the weight of the thymus, and increase the $\mathrm{T}$ - box type transcription factors (T-bet) and Th1 cytokines (IFN- $\gamma$, IL- 2 and IL12) expression level, reduce the PKC and intracellular $\mathrm{Ca} 2+$, ERK protein phosphorylation and p38 lightning activity, GATA-3 and Th2 cytokines (IL-4 and IL-10) expression level. Prove that wild jasmine glycoprotein from molecular level can effectively prevent the thymic atrophy and diethyl nitrosamine Th2 cytokines induced (Lee and Lim, 2012).

A novel glycoprotein isolated from Antrodia cinnamomea Mycelia was 442kD. It contained a particularly high content of uronic acid. The protein content was $71.0 \%$, apparently, higher than the carbohydrate content $14.1 \%$. It was confirmed to be totally non-detrimental to RAW 264.7 cell line even at dose $400 \mu \mathrm{g} / \mathrm{mL}$. It showed potent suppressing effect on the lipopolysaccharide induced inflammatory responses in RAW 264.7 cell line. The glycoprotein has revealed promising in vitro antiinflammatory effects (Chiu et al., 2014).

It was a glycoprotein $(27 \mathrm{kDa})$ isolated from Gardenia jasminoides Ellis (GJE) protects BNL CL.2 cells from expression of inflammation-related factors stimulated by cadmium chloride $(10 \mu \mathrm{M})$. Intracellular ROS and intracellular $\mathrm{Ca} 2+$ using fluorescence, activities of activator protein (AP)-1, cyclooxygenase (COX)-2, matrix metalloproteinase (MMP)-9, and arachidonic acid (AA) using immunoblot analysis or radioactivity were evaluated. The results obtained from this experiment indicated that GJE glycoprotein $(100 \mu \mathrm{g} / \mathrm{mL})$ inhibits the production of intracellular ROS, and intracellular $\mathrm{Ca} 2+$ mobilization. Also, it significantly suppressed inflammatory factors expression of AP-1 (c-Jun and c-Fos), arachidonic acid, COX-2, and MMP-9. (Lee and Lim, 2011).

\section{Natural Glycoprotein Cytotoxicity}

Glycoprotein cytotoxic effect on antitumor effect plays an important role, it directly kill tumor cells, block the cell cycle and tumor cell differentiation, activation of tumor necrosis factor, inhibition of tumor angiogenesis and metastasis. A $150 \mathrm{kDa}$ glycoprotein was isolated from Solanum nigrum can effectively inhibit the activity of human HCT-116 colon cancer cells and human breast cancer MCF-7 cells, and its mechanism may be used a dose-dependent inhibition of protein kinase PKC- $\alpha$, regulating nuclear factor NF- $\varkappa \mathrm{B}$ DNA binding activity, stimulation of intracellular NO production, suggesting that the plant Solanum glycoproteins can induce tumor cell apoptosis through regulatory mechanisms of cell signaling medium (Lee et al., 2004; Heo et al., 2005).

Seeds of Abrus isolated heterodimeric a $63 \mathrm{kDa}$ glycoprotein, and another $134 \mathrm{kDa}$ heterotetrameric glycoprotein, both glycoproteins have cytotoxicity, inhibit the synthesis pathway of tumor cells mainly proteins, are a potent inhibitor of protein synthesis, thereby inducing tumor cell death. Both glycoproteins by inhibiting synthesis of tumor cell growth in laboratory animals and the major protein component of the tumor cells, and enhance cell-mediated killing of tumor cells in the immune capacity, while in tumor-bearing animals also enhances the humoral immune response of the host, which activates its spleen cells and induce the body to produce Th1-type immune response, regulating tumorassociated macrophage-mediated tumor cell killing effect was significantly enhanced (Sujit et al., 2011).

Basilan isolated seed blooms out $27 \mathrm{kDa}$ glycoprotein or lectins, can inhibit the growth of MCF-7 human breast cancer cells, inhibition of laminin MCF-7 collagen and fibronectin adhesion, reduced $\alpha 1, \alpha 6$ and $\beta 1$ Asian integration expression group, increased expression of $\alpha 5$ subunit, inhibiting cell adhesion. Its anti-tumor effect by triggering tumor cell necrosis and secondary necrosis, inhibit the expression of caspase-9, leading to fragmentation of DNA fragments, resulting in decreased cell cycle arrest of $\mathrm{G} 2 / \mathrm{M}$ protein expression and regulation of p21 and $\mathrm{Rb}$ protein in the process to achieve (Mariana et al., 2014).

Functional properties of glycoprotein extracts from sweet potato (Ipomoea batatas LAM.) cultivars were conducted. The glycoprotein could inhibit the growth cells of COS-1, SHG-44 and SKOV3, the minimum dosage was $1.5 \mu \mathrm{g} / \mathrm{ml}$, according to in vitro tumor cell cultivation (Qian et al., 2005).

Sichuan pepper isolated from Zanthoxylum was $24 \mathrm{kDa}$ glycoprotein has antioxidant and anti-cancer effects, through the study of Balb/c mice hepatoma cells showed that the anti-tumor effect by increasing the Balb/c mice body piercing hormone secretory activity and granzyme $B$ and NK cells, inducing the expression of cytochrome C-related factors hepatocellular apoptosis and caspase-3, to promote apoptosis of tumor cells play a role in tumor suppression (Lee et al., 2014). Isolated from mushroom marmoreus out about the size of $52 \mathrm{kDa}$ glycoprotein U937 human leukemia cancer cells significantly inhibited. Marmoreus glycoprotein tumor effect by inhibiting cellular protein synthesis, and activation of immune cells in the tumor microenvironment responses, enhance the U937 tumor cytotoxic effect; inhibit cancer cell growth (Tsai et al., 2013).

Ricin, which is one of the most poisonous natural toxins known so far, is a plant 64000 glycoprotein extracted from the castor-oil plant. Using methoxy polyethylene glycol (MPEG) and polyethylene glycol (PEG) modified ricin was found to inhibit the synthesis of protein modifications at the same level, but lower 
cytotoxicity tripled on ricin head and neck cancer cells in the body have a certain effect of cancer, malignant melanoma, colon cancer, breast cancer, cervical cancer, and gastric cancer has a good effect (Lin et al., 1970; Pattrick et al., 2001; Suyalatu et al., 2010).

Separated from the brown seaweed kelp out of a glycoprotein that can inhibit a variety of tumor cells, such as gastric cancer cell AGS, HepG2 cells and colon cancer cells HT-29, added a dose-dependent inhibition of cancer cells, the tumor cell DNA fragmentation, Asia G1 arrest, caspase- 3 activation and PARP degradation, Fas and FADD-induced apoptosis caused, and procaspase-8 receptor induces the formation of signaling complexes. Show kelp glycoproteins by Fas signaling pathway, the mitochondrial pathway and cell cycle arrest mediated tumor cell apoptosis (Go et al., 2010). Kelp glycoprotein can not only inhibit tumor growth, but also enhance the spleen and thymus cancer U14 tumor-bearing mice, and increased with the $\mathrm{Bax} / \mathrm{Bcl}-2$ ratio of protein to induce tissue transplanted tumor cells apoptosis, performance a significant anti-tumor activity and low toxicity and activity (Zhai et al., 2013).

Seaweed pigment glycoprotein can promote the expression of Caspase- 3 and Bax protein, and induce the apoptosis of hepatoma cells. Seaweed pigment glycoprotein could significantly promote the expression of caspase- 3 and Bax, inhibit the proliferative activity and induce apoptosis of H22 hepatoma cells (Yang et al., 2011).

The glycoprotein obtained from the body wall of sea cucumber (Mensamaria intercedens) could significantly inhibit the growth of Sarcoma 180 cells implanted subcutaneously in mice at dosage of $20-30 \mathrm{mg} / \mathrm{kg}$ per day with no sign of toxicity from the whole body of sea cucumber (Mensamria intercedens), have shown good antitumor activity (Zou et al., 2003).

The glycoprotein groups are extracted and purified from crude Eupolyphaga sinensis Walker, which inhibits the growth of Eca109 and Hela cells remarkably at a low level dose $(<10 \mathrm{mg} / \mathrm{L})$. The results suggest that Eupolyphage sinensis Walker probably contains potent anti-tumor groups, which is extracted from the glycoprotein (Han et al., 2006).

The active glycoprotein-MTSGS1 from the mycelium of Tricholoma matsutake Sing. has antitumor activity in vitro. Low dose of MTSGS1 can inhibit cell proliferation and induced cell apoptosis by inhibiting cell conversion from S to G2M state. MTSG S1 inhibits S-180 cell multiplication in vivo cell, the antitumor ratio is $65 \%$ at $50 \mathrm{mg} / \mathrm{kg}$ per day. The spleen weight and index increase markedly. The results show that MTSGS1 has obvious antitumor activity in vitro and in vivo (Liu et al., 2001).

Tricholoma madsutake mycelium glycoprotein, molecular $23.7 \mathrm{kDa}$, it has a direct cytotoxic effect in MCF-7 cells, within a certain range of concentrations showing dose and time-dependent, increasing with the dose and duration of action prolonged inhibition of cell rate increases, breast cancer cells in vitro with good direct killing effect on normal liver cells are less toxic (Wei et al., 2005).

In the two isolated mistletoe inhibit tumor cell growth
Loranthaceae glycoprotein, molecular weight of about 31,000 and 33,000, with inhibition of hepatic tumor cells Bel-7402 the growth of, IC50 value of $6 \mathrm{mg} / \mathrm{L}$. Loranthaceae glycoproteins proved a significant inhibition of tumor (Zhang et al., 2006).

Momordicin is extracted from the seeds of Momordica charantia L. gourd vegetable commonly used out of the active ingredient, is a molecular weight of about $30 \mathrm{kDa}$ basic glycoprotein Momordicin has a good anti-tumor effect, no toxic effects on normal cells. Momordicin can reverse human chronic myeloid leukemia cell lines was inhibited apoptosis of K562/A02, the main mechanism may be associated with reduced expression of p53, P-gp and bcl-2 and the increase caspase family activity, and promote apoptosis. Momordicin can induce apoptosis in leukemia cells resistant to reverse tumor cell MDR, and normal cultured cells no significant adverse reactions (Yin et al., 2006).

\section{Glycoprotein Inhibit Endothelial Growth Factor}

Growth and transfer of tumor cells, depends largely on the vascular endothelial cell growth factor, how to control the activity of the expression of endothelial factor, specific angiogenic factors and related factors of relationship between tumors and factors, based on the research of glycoprotein biology found that glycoprotein can effectively inhibit endothelial cell growth factor and antitumor effect (Feng et al., 2014; Kumar et al., 2014).

Neem leaf glycoprotein (NLGP) prevention and therapeutic drug induced solid tumor growth in mice significantly. NLGP can make the cancer and melanoma blood vessels in the normalization, the expression of vascular endothelial growth factor and VEGFR2 cut, can adjust the VEGF-VEGFR-2 pathway of vascular endothelial cell signal transduction, to prevent abnormal new blood vessels to form. Limited NLGP active in tumor growth and the process of tumor angiogenesis play an important role (Banerjee et al., 2014).

Isolated from plants in India squill bulbs out a novel glycoprotein $29 \mathrm{kDa}$, with potent against ascites tumor and mouse mammary tumor growth, and its anti-tumor mechanism of inhibition of tumor angiogenesis and inhibition of proliferation in vitro by human umbilical vein endothelial cells (HUVEC) inhibition experiments showed that glycoprotein induced by vascular endothelial growth factor in vitro inhibition of proliferation, further supporting its anti-angiogenic activity. Induced nuclear factor NF- $\varkappa \mathrm{B}$ translocation and activation of caspase-3 expression in tumor cells, in vivo induction of DNA breaks, prove the glycoprotein has caused inhibition of tumor angiogenesis and expression of vascular endothelial growth factor gene declining role (Deepak et al., 2006).

An angiogenesis inhibitor isolated from shark cartilage, SCF2, has been characterized. SCF2 was shown to have specific angiogenesis-inhibiting activity in endothelial cell culture assays. Structural and functional studies indicate that the inhibitor is a heat-stable proteoglycan, which contains keratan sulfate units and peptide. Gel filtration chromatography shows that the molecular weight of the 
angiogenesis inhibitor is about $10 \mathrm{kd}$. Thresher Sharks angiogenesis thereby inhibiting tumor growth (Liang et al., 2000).

Tumor-associated macrophage-like phenotype and M2 lead to abnormal tumor vasculature by macrophages produce angiogenic factors such as the PIGF and M2cytokines such as IL10 and CCL22. M2- cytokines inhibit immune cell function. histidine-rich glycoprotein (HRG) increased levels of differentiated macrophage-like phenotype away from the M2 normalization of tumor vessels and activates antitumor immunity. Through enhancing hemangioma, normalize blood vessels and may reduce metastatic tumor cells, may also promote the drug absorption and the activity of immune cells. it may promote drug absorption and immune cell activit, reduce the value of the pressure sensitivity of malignant tumor cloning and promote M1 class TAM phenotype. HRG treatment may result in reduced tumor growth and metastasis, and increased the effectiveness of various therapies (Huang et al., 2011).

\section{Conclusions}

Glycoprotein through a variety of different mechanisms to suppress tumor cells, and study the relationship between the activity and function of the mechanism glycoprotein between tumor cells and will be further deepened. Natural glycoprotein set low toxicity, safety and widely studied and applied in a variety of disciplines, such as medicine and health food products are increasingly being used in the field, give full play to the anti-tumor effect of natural glycoprotein for clinical finding safer and more effective drugs tumor killing and suppressing drugs to provide effective avenues of research.

Glycobiology research focuses on the gradual shift to resolve its role in diseases such as the direction of the sugar chain structure. At present, the resolution and determination of glycoprotein structure have methods such as capillary electrophoresis, high performance liquid chromatography (HPLC). Mainly through fixed point mutation of glycoprotein functions of constructing single glycosylation sites knockout plasmid transfection into tumor cell lines, the glycoprotein structure in different glycosylation sites lack of influence on its function, more accurate to determine the effect of sugar chain of glycoprotein function; In addition, through specific glycosyl transferase or application glycosyl transferase inhibitor expressed in cell lines to confirm the importance of the specific function of sugar chain structure of glycoprotein (Wang et al., 2012).

Use of glycoprotein structural diversity and unique biological activity, its significant anti-tumor activity and good bioavailability seek active glycoprotein macromolecules, their structure, from the level of molecular mechanisms to explain its role in tumor research and analysis through modern technology mechanism. At present, the use of a biologically active glycoprotein design antitumor agents, to stimulate the immune system for therapeutic purposes. The results of drug development for the treatment of malignant tumor and its related provides research basis.

\section{Acknowledgements}

This research is supported by Harbin University of Commerce Natural Science Foundation of Young Teachers, Heilongjiang Province (No: HCUL2013019) and (No: HCUL2013018).

\section{References}

Andrews P, Zhao X, Allen J, et al (2008). A comparison of the effectiveness of selected non-steroidal anti-inflammatory drugs and their derivatives against cancer cells in vitro. Cancer Chemother Pharmacol, 61, 203-14

Barik S, Bhuniya A, Banerjee S, et al (2013). Neem leaf glycoprotein is superior than Cisplatin and Sunitinib malate in restricting melanoma growth by normalization of tumor microenvironment. Int Immunopharmacol, 17, 42-9.

Banerjee S, Ghosh T, Barik S, et al (2014). Neem leaf glycoprotein prophylaxis transduces immune dependent stop signal for tumor angiogenic switch within tumor microenvironment. PLoS One, 9, 110040.

Bose A, Chakraborty K, Sarkar K, et al (2009). Neem leaf glycoprotein directs T-bet-associated type 1 immune commitment. Hum Immunol, 70, 6-15.

Chiu CH, Peng CC, Ker YB, et al (2014). Physicochemical characteristics and anti-inflammatory activities of antrodan, a novel glycoprotein isolated from Antrodia cinnamomea mycelia. Molecules, 19, 22-40.

Dai L, Wang H, Chen Y (2000). The immune-enhancing effect of PcG-A-a glycoprotein isolated from dried root of pulsatilla chinensis (bunge) regel. Chin J Biochemical Pharmaceutics, 21, 230-1.

Das A, Barik S, Banerjee S, et al (2014). A monoclonal antibody against neem leaf glycoprotein recognizes carcinoembryonic antigen (CEA) and restricts CEA expressing tumor growth. J Immunother, 37, 394-406.

Deepak AV, Salimath BP(2006). Antiangiogenic and proapoptotic activity of a novel glycoprotein from $U$. indica is mediated by NF-kB and caspase activated DNase in ascites tumor model. Biochimie, 88, 297-307.

Feng Y, Gross S, Wolf NM, et al (2014). Nucleoside diphosphate kinase $\mathrm{B}$ regulates angiogenesis through modulation of vascular endothelial growth factor receptor type 2 and endothelial adherens junction proteins. Arterioscler Thromb Vasc Biol, 34, 2292-300.

Goswami KK, Barik S, Sarkar M, et al (2014). Targeting STAT3 phosphorylation by neem leaf glycoprotein prevents immune evasion exerted by supraglottic laryngeal tumor induced M2 macrophages. Mol Immunol, 59, 119-27.

Go H, Hwang HJ, Nam TJ (2010). A glycoprotein from Laminaria japonica induces apoptosis in HT-29 colon cancer cells. Toxicol in Vitro, 24, 1546-53.

Grigorian A, Torossian S, Demetriou M (2009). T-cell growth, cell surface organization, and the galectin glycoprotein lattice. Immunol Rev, 230, 232-46.

Gupta S, Zhang D, Yi J, et al (2004). Anticancer activities of Oldenlandia diffusa. J Herb Pharmacother, 4, 21-33.

Gu QQ, Fang YC, Xin XL, et al (2001). Effects of glycoprotein from Chlamys farreri on tumor growth and immune function in mice. Acta Nut Sin, 23, 200-2.

Han YL, Xie K (2006). The glycoprotein group extracted and purified from Eupolyphaga sinensis walker inhibits the growth of tumor cell in vitro. J Shantou Univ (Nat Sci), 21, 46-50.

Huang Y, Snuderl M, Jain RK (2011), Polarization of tumor- 
associated macrophages: a novel strategy for vascular normalization and antitumor immunity. Cancer Cell, 19, 1-2.

Hanahan D, Weinberg RA (2011). Hallmarks of cancer: the next generation. Cell, 144, 646-74.

Heo KS, Lim KT (2005). Glycoprotein isolated from Solanum nigrum L. modulates the apoptotic-related signals in 12-O-tetradecanoylphorbol 13-acetate-stimulated MCF-7 cells. J Med Food, 8, 69-77.

Kumar VB, Binu S, Soumya SJ, et al (2014). Regulation of vascular endothelial growth factor by metabolic context of the cell. Glycoconj J, 31, 427-34.

Lee J, Lim KT (2012). Inhibitory effect of Styrax Japonica Siebold, Zuccarini glycoprotein (38kDa) on interleukin- $1 \beta$ and induction proteins in chromium (VI)-treated BNL CL.2 cells. Mol Cell Biochem, 367, 103-11.

Lee J, Lim KT (2012). SJSZ glycoprotein (38kDa) prevents thymus atrophy and enhances expression of IL-2 and IL-12 in diethylnitrosamine-induced hepatocarcinogenesis. Int Immunopharmacol, 13, 362-9.

Lee J, Lee SJ, Lim KT (2014). ZPDC glycoprotein (24kDa) induces apoptosis and enhances activity of NK cells in $\mathrm{N}$-nitrosodiethylamine-injected Balb/c. Cell Immunol, 289, 1-6.

Lee SJ, Oh PS, Ko JH, et al (2004). A 150-kDa glycoprotein isolated from Solanum nigrum L. has cytotoxic and apoptotic effects by inhibiting the effects of protein kinase $\mathrm{C}$ alpha, nuclear factor-kappa B and inducible nitric oxide in HCT-116 cells. Cancer Chemother Pharmacol, 54, 562-72.

Liang JH, Wong KP (2000). The characterization of angiogenesis inhibitor from shark cartilage. Adv Exp Med Biol, 476, 209-23.

Liu P, Tao WY, Sun ZH, et al (2001). The antitumor effect and mechanism of active glycoprotein MTSGS1 from tricholoma matsutake mycelium. Pharm Biotechnol, 8, 284-7

Lin JY, Tserng KY, Chen CC (1970). Abrin and ricin: new antitumor substances. Nature, 227, 292-3.

Lee J, Lim KT (2011), Inhibitory effect of plant-originated glycoprotein $(27 \mathrm{kDa})$ on expression of matrix metalloproteinase-9 in cadmium chloride-induced BNL CL. 2 cells. J Trace Elem Med Biol, 25, 239-46.

Mariana CC. Silva, Claudia AA. de Paula, et al (2014). Bauhinia forficata lectin (BfL) induces cell death and inhibits integrinmediated adhesion on MCF7 human breast cancer cells. Biochim Biophys Acta, 1840, 2262-71.

Mantovani A, Allavena P, Sica A, Balkwill F (2008). Cancerrelated inflammation. Nature, 454, 436-44.

Nie SP, Xie MY, Fu ZH, et al (2008). Study on the purification and chemical compositions of tea glycoprotein. Carbohyd Polym, 71, 626-33.

Nie SP, Xie MY, Zhou P, et al (2007). In vitro antioxidative and anticancer activities of tea glycoprotein in green tea. Eur Food Res Technol, 224, 437-42.

Pattrick G, Richardson SW, Casolaro M, et al (2001). Poly (amdoamine) mediated intracytoplasmic delivery of ricin A-chain and gelonin. J Control Release, 77, 225-33.

Pandurangan AK, Dharmalingam P, Ananda Sadagopan SK, et al (2012). Effect of luteolin on the levels of glycoproteins during azoxymethane-induced colon carcinogenesis in mice. Asian Pac J Cancer Prev, 13, 1569-73.

Qian JY, Liu D, Sun HC (2005). Study on functional properties of sweet potato glycoprotein-an in vitro antitumor and ames tests. Food Sci, 26, 216-8 (in Chinese).

Roy S, Barik S, Banerjee S, et al (2013). Neem leaf glycoprotein overcomes indoleamine 2,3 dioxygenase mediated tolerance in dendritic cells by attenuating hyperactive regulatory $\mathrm{T}$ cells in cervical cancer stage IIIB patients. Hum Immunol, 74, 1015-23.
Sandra D, Eli P, Michael K, et al (2010). Cancer and inflammation: promise for biological therapy. J Immunother, 33, 335-51.

Sujit K. Bhutia, Tapas K. Maiti (2011). Chapter 49-Crabs Eye (Abrus precatorius) Seed and Its Immunomodulatory and Antitumor Properties. Nuts and Seeds in Health and Disease Prevention, 409-15.

Suyalatu, Huang FL, Zhang JX, et al (2010). Research Progress of Ricin, J Inner Mongolia Univ Nat, 25, 419-22.

Takahashi M, Kuroki Y, Ohtsubo K, et al (2009). Core fucose and bisecting GlcNAc, the direct modifiers of the N-glycan core: their functions and target proteins. Carbohydr Res, 344, 1387-90.

Tsai PF, Ma CY, Wu JS (2013). A novel glycoprotein from mushroom Hypsizygus marmoreus (Peck) Bigelow with growth inhibitory effect against human leukaemic U937 cells. Food Chemistry, 141, 1252-8.

Wang HY, Wu LJ, Yuan Y, et al (2012). Advances on the antitumor effects of glycoprotein and its mechanism. Ningxia $J$ Agri Fores, 53, 119-21.

Wei YQ, Xu HY, Xu ZH, et al (2005). Inhibition effect of glycoprotein MTS03 from the submerged mycelia of tricholoma madsutake on proliferation in MCF-7 cells in vitro. Chin Pharm J, 40, 1545-8.

Wu PK, Chi Shing Tai W, Liang ZT, et al (2009). Oleanolic acid isolated from Oldenlandia diffusa exhibits a unique growth inhibitory effect against ras-transformed fibroblasts. Life Sci, 85, 113-21.

Yang Q, Zhong JY, Li S, et al (2011). Effects of seaweed pigment glycoprotein on caspase-3 and bax protein expression of hepatoma cells. Progress Modern Biomedicine, 11, 1771-4.

Yin LH, Xiong SD, Ye AF, et al (2010). Effect of momordin in inhibiting proliferation and inducing apoptosis of multidrugresistant K562/A02 cells and its molecular mechanism. Tumor, 30, 288-91.

Zhai Q, Li X, Yang Y, Yu L, et al (2014). Antitumor activity of a polysaccharide fraction from Laminaria japonica on U14 cervical carcinoma-bearing mice. Tumour Biol, 35, 117-22.

Zhang LS, Liu SL (2006). Isolation of anticancer proteins from lorathlorace and part property studying. Nat Prod Res Dev, 18, 43-6.

Zou Z, Yi Y, Wu H. Wu J, Liaw C, Lee K (2003). Intercedensides A-C, three new cytotoxic triterpene glycosides from the sea cucumber Mensamaria intercedens Lampert. J Nat Prod, 66, 1055-60. 